\title{
Postural stabilization during bilateral and unilateral vibration of ankle muscles in the sagittal and frontal planes
}

Noémie C Duclos ${ }^{1 *}$, Luc Maynard², Joëlle Barthelemy ${ }^{1}$ and Serge Mesure ${ }^{1}$

\begin{abstract}
Background: The purpose was to investigate the postural consequences of proprioceptive perturbation of the Triceps Surae and Peroneus Longus muscles. These muscles are known to control posture respectively in the sagittal and frontal planes during standing.

Methods: Standard parameters and the time course of center of pressure (COP) displacements were recorded in 21 young adults, instructed to maintain their balance during tendon vibration. Following $4 \mathrm{~s}$ of baseline recording, three types of vibration $(80 \mathrm{~Hz}$ ) were applied for $20 \mathrm{~s}$ each on the Peroneus or Achilles tendons, either unilaterally or bilaterally (with eyes shut). The recording continued for a further $24 \mathrm{~s}$ after the end of the vibration during the re-stabilization phase. To evaluate the time course of the CoP displacement, each phase of the trial was divided into periods of 4 seconds. Differences between the type of tendon vibration, phases and periods were analyzed using ANOVA.
\end{abstract}

Results: During all tendon vibrations, the speed of the CoP increased and a posterior displacement occurred. These changes were greater during Achilles than during Peroneus vibration for each type of vibration and also during bilateral compared with unilateral vibration. All maximal posterior positions occurred at a similar instant (between 12.7 and $14 \mathrm{~s}$ of vibration). Only unilateral Achilles vibration led to a significant medio-lateral displacement compared to the initial state.

Conclusions: The effect of the proprioceptive perturbation seems to be influenced by the position of the vibrated muscle according to the planes of the musculoskeletal postural organization. The amplitude of the destabilization may be related to the importance of the muscle for postural control. The medial CoP displacement which occurred during unilateral Achilles vibration is not a general reaction to a single-limb perturbation. Proprioceptive input from the non-perturbed leg was not sufficient for the antero-posterior displacement to be avoided; however, it helped to gain stability over time. The non-perturbed limb clearly plays an important role in the restoration of the postural referential, both during and immediately following the end of the vibration. The results demonstrated that at least $16 \mathrm{~s}$ of vibration are necessary to induce most postural effects in young adults.

Keywords: Proprioception, Plan-dependence, Postural control, Standing position, Unilateral vibration

\section{Background}

The role of the postural control system is to continuously manage the body's state of equilibrium in order to avoid falls. Balance is constantly perturbed by body and limb motion and the postural control system must select and integrate relevant sensory information in order to

\footnotetext{
*Correspondence: noemie.duclos@univ-amu.fr

'Aix-Marseille Université, CNRS, ISM UMR 7287, 13288 Marseille, France Full list of author information is available at the end of the article
}

maintain postural control [1]. Upright postural behavior is comparable to an inverted pendulum [2]. The perturbation of one source of sensory information will result in different consequences depending on the pertinence of that information for the postural requirements at that instant [3]. Postural control in the sagittal plane predominantly occurs at the ankle [4], with the Soleus muscle playing a major role in keeping the body upright and controlling antero-posterior oscillations [5]. In contrast, in the frontal 
plane, postural control mainly occurs at the hip level [6]. However, it has been shown that control also occurs at the ankle, with the Peroneus Longus providing lateral stability [7]. During normal upright standing, proprioceptive information from the legs provides the most pertinent source of afferent information for the control of postural sway [8]. Most studies of proprioception around the ankle joint have focused on the sagittal plane (for example $[9,10]$ ) and the frontal plane has been little explored. This is probably because of the mechanical stability naturally provided by the distance between the two feet in normal standing [11]. Nevertheless, a deficit of proprioceptive capacity could explain the medio-lateral instability [12] typically found in older adults, which is considered to be the best predictor of the risk of falls [13].

Many studies have used sudden movements of the supporting surface to explore the role of the ankle in postural reactions in both planes of motion [14]. There are two problems with the use of this paradigm: (i) the amplitude of destabilization often generates hip strategies rather than ankle strategies and (ii) the perturbation is applied bilaterally whilst the postural challenges which occur during daily activities are often unilateral. Thus, in order to better understand the role of the ankle in postural stability, it is necessary to explore the role of proprioception (i) during both unilateral and bilateral perturbations (ii) from ankle muscles which act predominantly in the sagittal and frontal planes. Tendon vibration is a useful tool for this purpose because it can be used to perturb proprioception in specific muscles [15]. The vibration principally activates primary (Ia) afferents in the muscle spindles [16], mimicking muscle stretch [15]. When the vibration is applied at $80 \mathrm{~Hz}$ [17] to a subject in quiet standing, the vibration generates an afferent signal [18] and a postural reaction occurs to 'restore' muscle length and avoid the illusory fall. The amplitude of the postural reaction reflects the integration of this signal in the postural scheme.

Most studies have evaluated the effect of bilateral Achilles vibration (for example $[19,20]$ ), showing that it results in a backward shift of the CoP. The few studies which have applied Achilles vibration to a single limb [21,22] have shown that a medial displacement of the CoP also occurs. However, the underlying mechanisms have not been explored. The medial displacement could be a general response to the perturbation of a single limb, irrespective of the tendon targeted (Achilles or Peroneus longus). Moreover, during bilateral vibration, the CoP shift gradually reduced as the vibration continued [20]. Questions however remain regarding the time course of the CoP displacement as well as regarding the specific role of the non-vibrated limb. It remains to be determined whether its role is to counteract the effect of the vibration or to regulate the postural reaction.
The aims of this study were threefold. The first aim was to determine if the postural reaction depends on the plane of motion controlled by the perturbed muscle. The second aim was to determine the role of the non-perturbed limb as a function of the direction of the induced reaction. The third aim was to investigate the time course of the CoP displacement during and following the perturbation in the different vibration conditions.

\section{Methods \\ Participants}

21 young-adults (11 women/10 men; 24.0 (4.83) [mean (SD)] years old, 69.3 (10.8) $\mathrm{kg}$ and 1.75 (0.08) m; dominant hand: 17 right/4 left; dominant eye: 14 right/7 left; dominant foot: 8 right/13 left) were included. All participants signed an informed consent form prior to the study. The study was conducted in accordance with the Declaration of Helsinki and approved by the Local Ethics Committee Sud-Méditerranée II (nID-RCB 2012-AOO518-35).

\section{Apparatus}

Each subject was asked to stand quietly barefoot on the force platform, with their feet in a natural position (Figure 1; [23]) and one foot on each side of the AP axis reference mark on the force plate. Vibrations were carried out on the tendons of two muscles: peroneus longus (just above the lateral malleolus, with additional manual palpation; [24]); and gastrocnemius-soleus (at the level of the lateral malleolus). Subjects were instructed to maintain their balance at all times.

\section{Experimental protocol}

Foot positions were marked on the force plates in order to ensure constant placement throughout the experiment. Three types of vibration (each repeated twice) were carried out: left unilateral, right unilateral and bilateral. Site order and trial sequences were randomized. Subjects wore opaque glasses to block vision for all trials. They were asked to keep their gaze in a straightahead direction. Unless they needed to rest, the subjects did not remove the opaque glasses for the duration of the experiment.

No vibration was applied for the first 4 seconds of each trial (P1 phase); vibration was then applied for 20 seconds (P2 phase; [25]) and the recording was continued for a further 24 seconds after the end of the vibration during re-stabilization (P3 phase). A DC motor with an eccentric load on the shaft, embedded in a plastic tube which was $7 \mathrm{~cm}$ long and 2,5 cm wide (VB 115, TechnoConcept, Mane, France), was used. Vibration frequency was set at $80 \mathrm{~Hz}$ with an amplitude of 0.2-0.5 mm [17]. These parameters are considered to be the most pertinent for the generation of illusory movement according to Roll et al. [15-17]. 


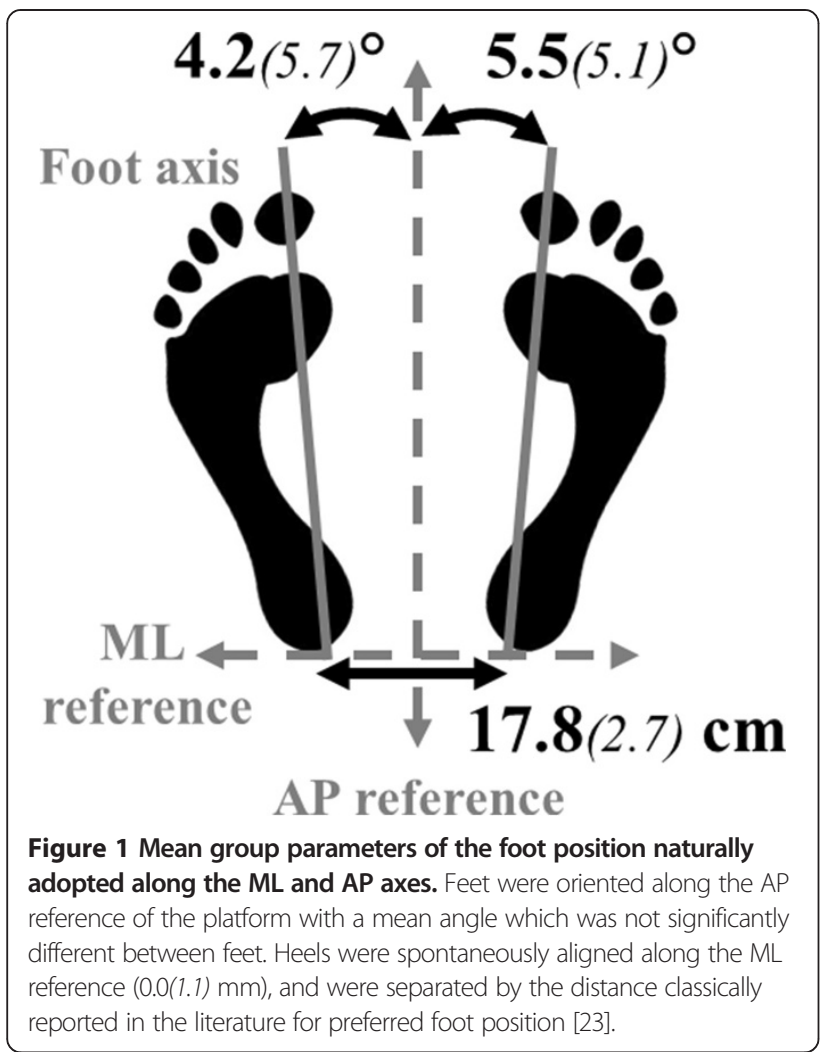

\section{Data acquisition}

Ground reaction forces were calculated from 8 vertical mono-axial dynamometric load sensor cells; at a frequency of $40 \mathrm{~Hz}$. The acquisition of the ground reaction forces was synchronized with the stimulus. Force data were then processed using Matworks's MATLAB v.6 software to calculate postural parameters.

\section{Postural parameters}

Antero-posterior (AP) and medio-lateral (ML) displacement of the CoP were recorded. The following parameters were then calculated for each phase of each trial:

- Speed of the CoP displacement, calculated as total excursion of the COP divided by the duration, in each phase.

- Mean anterio-posterior and medio-lateral CoP positions $(\mathrm{mm})$; Negative values represent a posterior or left position relative to the center of the platform.

- Maximum and minimum positions ( $\mathrm{mm}$ ) along the antero-posterior axis (AP-max and AP-min) and their time of occurrence (s).

To compare positions across trials, the mean position of the CoP at P1 was centered on zero for both axes by subtracting its coordinates from all the data samples recorded throughout the trial.

\section{Time course of the CoP}

To evaluate the time course of the CoP displacement, each phase of the trial was divided into periods of 4 seconds.
The vibration phase, P2 (20 s) was divided into 5 periods ( $\mathrm{P} 2_{1}$ through $\mathrm{P} 2_{5}$ ). The last phase, P3 (re-stabilisation, 24 s) was divided into 6 periods ( $\mathrm{P} 3_{1}$ through $\left.\mathrm{P} 3_{6}\right)$.

\section{Statistical analysis}

After verifying the normality of the data, all parameters were analyzed using a general linear model repeated measures of variance analysis (ANOVA). Differences between the Achilles and Peroneus tendon vibration were analyzed using ANOVA with three within-subject factors: "Tendon" (Achilles, Peroneus), "Type” (bilateral, right unilateral, left unilateral) and "Phase" (P1, P2, P3). Postural adaptations relative to the initial state and over time were analyzed for each tendon using ANOVA with two within-subject factors: "Type" and "Period" (P1/P2 $2_{1}-\mathrm{P} 2{ }_{5}$, then $\left.\mathrm{P} 1 / \mathrm{P} 3_{1}-\mathrm{P} 3_{6}\right)$. A parametric Newman-Keuls post hoc test was used to determine the locus of differences. An $\alpha$ level of 0.05 was used for all tests.

\section{Results}

All subjects accomplished the task without falling. There were no significant differences between conditions for the parameters analyzed during the P1 phase. In the text, "unilateral vibration" refers to both right and left unilateral vibrations, unless otherwise specified.

\section{Effect of the tendon vibrated}

There was a Tendon $\times$ Type $\times$ Phase interaction for all parameters (Table 1). CoP displacements were perturbed during the vibration and also when it stopped, depending on the tendon and the type of vibration.

\section{Vibration phase (P2)}

All tendon vibrations led to an increase in the speed and a posterior displacement of the CoP. This effect was significantly greater during Achilles than Peroneus vibration $(\mathrm{p}<0.01)$ for each type. For both tendons, the speed and the posterior displacement were larger during bilateral than unilateral vibration ( $\mathrm{p}<0.01$; Figure 2 ). Only unilateral Achilles vibration led to a significant ML displacement compared to the initial state (Figure 2).

The AP-max position (anterior to zero) was similar for all conditions of vibration. The AP-min position was significantly more posterior for Achilles than Peroneus vibration ( $p<0.01$ for each condition) and it was also more posterior for bilateral than unilateral vibration ( $\mathrm{p}<0.01$; Figure 3$)$. The AP-min position during right and left unilateral Achilles vibration was respectively $49.42 \%$ (18.22) and $54.38 \%$ (22.64) of the AP-min during bilateral vibration. The AP-min position during right and left unilateral Peroneus vibration was respectively $51.76 \%$ (20.55) and $63.74 \%$ (23.87) of the AP-min during bilateral vibration. 
Table 1 ANOVA results for both Achilles and Peroneus tendon vibrations

\begin{tabular}{lcccc}
\hline Parameters & Tendon effect & Type effect & Phase effect & Tendon $\times$ Type $\times$ Phase interaction \\
\hline Speed & $F(1,20)=54.2$ & $F(2,40)=104.0$ & $F(2,40)=2.8$ & $F(4,80)=7.2$ \\
AP position & $S$ & $S$ & $S$ & $S$ \\
& $F(1,20)=10.3$ & $F(2,40)=8.0$ & $F(2,40)=80.5$ & $S$ \\
ML position & $S$ & $S$ & $F(2,40)=0.7$ & $S$ \\
& $F(1,20)=0.9$ & $F(2,40)=16.0$ & $p=0.5$ & $F(4,80)=17.0$ \\
\hline
\end{tabular}

ANOVA results showing the effect of 'Tendon' (Achilles/Peroneus Longus), 'Type' (bilateral/left unilateral/right unilateral), 'Phase' (P1: stable/P2: vibration/P3: restabilisation) and their interactions. Significant results are written in bold text. $S=$ statistically significant $(p<0.05)$.

Time course of the CoP during vibration (P1/P2 $\left.1-P 2_{5}\right)$

There was a main effect of Type and Period on mean CoP position along the AP axis during both Achilles and Peroneus vibrations and also a Type $\times$ Period interaction (Table 2).

Mean CoP position shifted significantly posteriorly from $\mathrm{P} 2{ }_{1}$ through $\mathrm{P} 2{ }_{4}$ for bilateral and unilateral left Achilles vibration (Figure 4), then it stabilized. For unilateral right Achilles vibration, the mean position only shifted significantly posteriorly from $\mathrm{P} 2{ }_{1}$ to $\mathrm{P} 22_{2}$.

Mean CoP position also shifted significantly posteriorly from $\mathrm{P} 21_{1}$ through $\mathrm{P} 24_{4}$ for bilateral Peroneus vibration. During unilateral left Peroneus vibration, the mean CoP position only shifted posteriorly from $\mathrm{P} 2_{1}$ to $\mathrm{P} 2_{2}$ and during right Peroneus vibration there was no change from the mean $\mathrm{P} 22_{1}$ position.

There was a main effect of Type and Period, and a Type $\times$ Period interaction for the mean medio-lateral
CoP position during Achilles vibrations only (Table 2). The position shifted significantly medially across all the periods of P2 during left unilateral vibration whereas it stabilized from $\mathrm{P} 2_{2}$ in the right unilateral vibration (Figure 5).

There was a main effect of Type but no effect of Period (Table 2) on CoP speed during both Achilles and Peroneus vibration. For bilateral Achilles and Peroneus vibrations, the speed of the CoP increased significantly from P1 to P2 and from $\mathrm{P} 21_{1}$ to $\mathrm{P} 2_{2}$ (Figure 6). For all unilateral Achilles and Peroneus vibrations, the speed increased from P1 to $\mathrm{P} 2{ }_{1}$ only, then stabilized until the end of vibration.

The AP-max occurred at a similar instant (between $2.35 \mathrm{~s}$ and $4.8 \mathrm{~s}$ of vibration) for all conditions of vibration (except for right unilateral Peroneus). All AP-min positions occurred at a similar instant (between 12.7 and $14 \mathrm{~s}$ of vibration, ie. in the $\mathrm{P} 24$ period) regardless of the tendon or type of vibration (Figure 3 ).

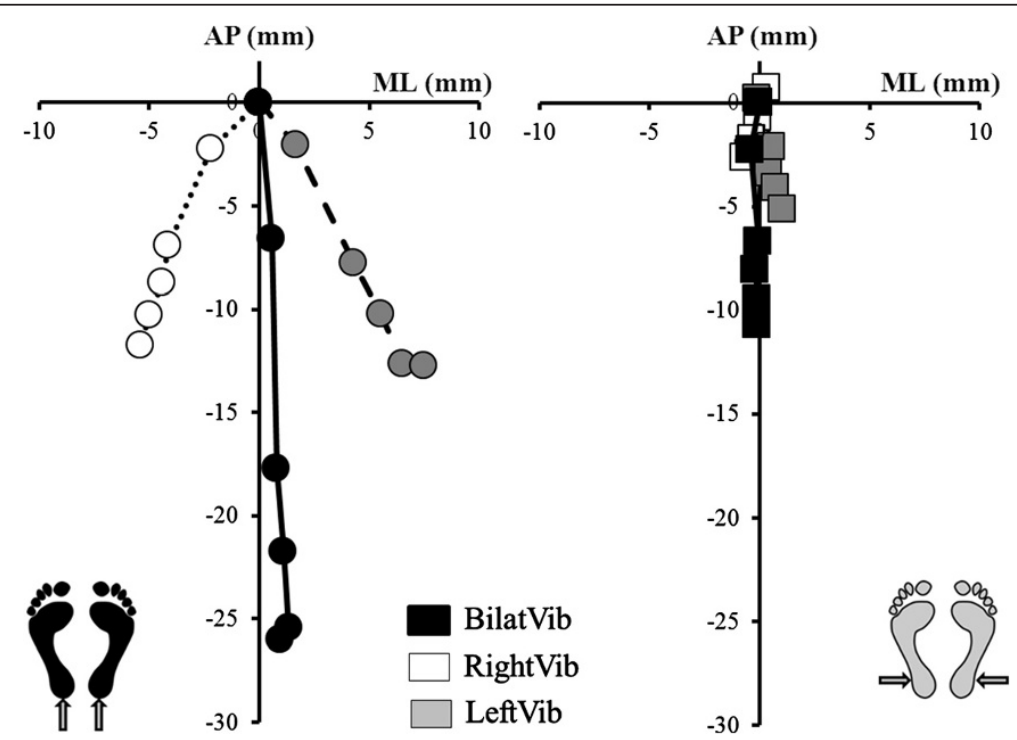

Figure 2 Antero-posterior and medio-lateral positions during Achilles and Peroneus vibration. Representation of AP (antero-posterior) and $\mathrm{ML}$ (medio-lateral) coordinates of the mean positions for the 5 periods of the vibration-phase (P2), during [left] Achilles (circles) and [right] Peroneus (squares) vibration, bilateral (black squares); right unilateral (white squares) and left unilateral type (grey squares). ML displacement was significant for unilateral Achilles vibrations only. 


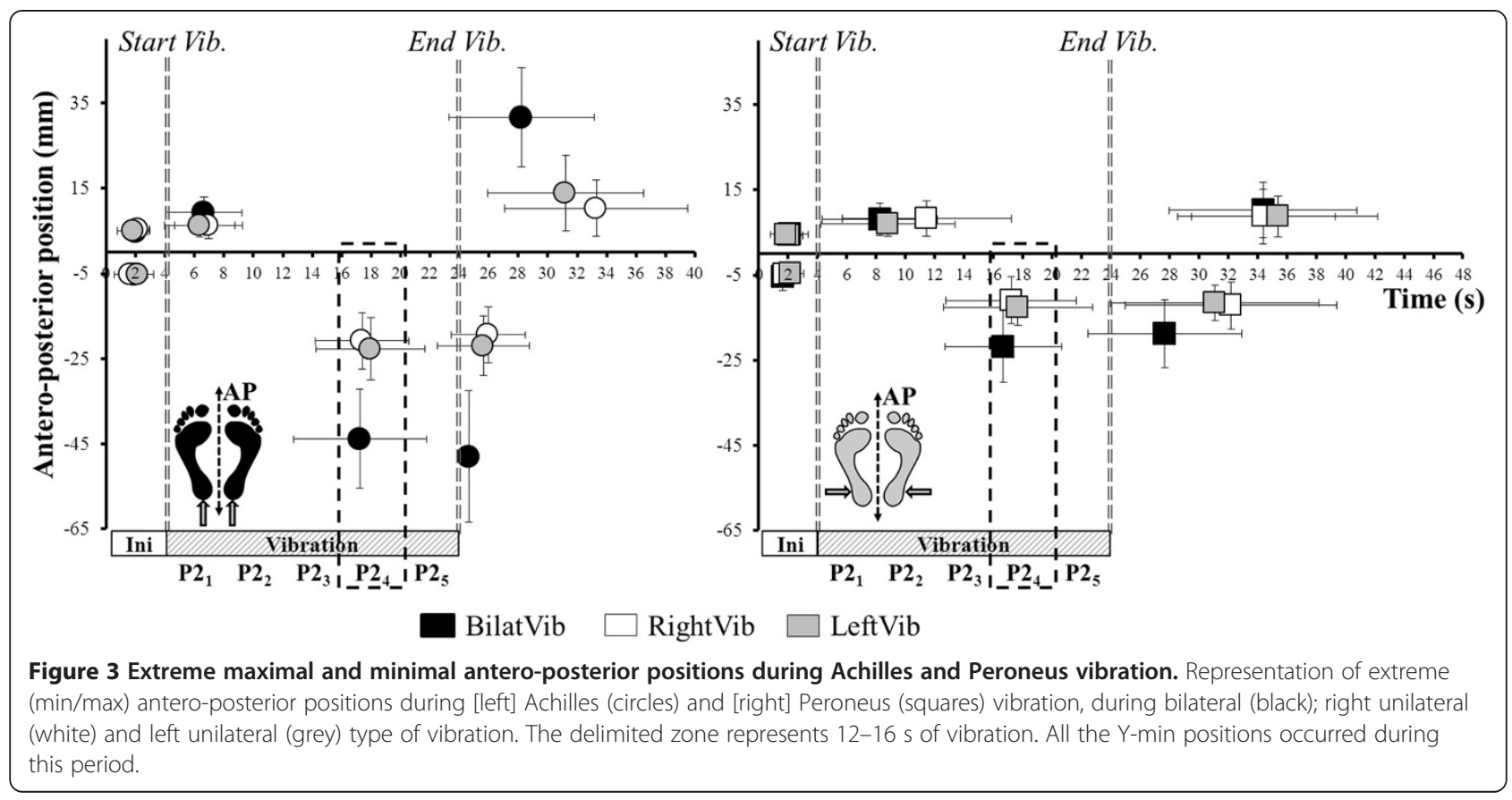

Post-effect of vibration (P1/P3 $1-\mathrm{P}_{6}$ )

P3 postural parameters were not averaged because they were affected by the irremediable return to the initial state. The parameters were thus analyzed by periods $\left(\mathrm{P} 3_{1}\right.$ through P36). There was a main effect of Period (Table 2) for all parameters except the ML-position of the CoP. After bilateral Achilles vibration, the mean AP-position remained anterior to zero until the end of the trial. In contrast, after cessation of unilateral Achilles vibration, the CoP returned to its initial position on the AP-axis almost immediately. Mean ML-position, however, differed from zero until $\mathrm{P}_{3}$ after left Achilles vibration and until the end of the trial after right vibration.

After all Peroneus vibrations, mean AP-position returned close to zero at $\mathrm{P}_{2}$ and mean ML-position did not differ from zero, as was the case during the vibration phase.

Regardless of the tendon vibrated, the speed of the CoP returned close to the initial state at $\mathrm{P} 3_{3}$ after

Table 2 ANOVA results for Achilles and Peroneus tendon vibrations separately

\begin{tabular}{|c|c|c|c|c|c|c|}
\hline Parameters & $\begin{array}{c}\text { Type effect } \\
\left(\mathrm{P} 1 / \mathrm{P} 2_{1}-\mathrm{P} 2_{5}\right)\end{array}$ & $\begin{array}{l}\text { Period effect } \\
\left(\mathrm{P} 1 / \mathrm{P} 2_{1}-\mathrm{P} 2_{5}\right)\end{array}$ & $\begin{array}{c}\text { Type } \times \text { Period } \\
\left(\mathrm{P} 1 / \mathrm{P} 2_{1}-\mathrm{P} 2_{5}\right) \\
\text { interaction }\end{array}$ & $\begin{array}{c}\text { Type effect } \\
\left(\mathrm{P} 1 / \mathrm{P3} 3_{1}-\mathrm{P3}_{6}\right)\end{array}$ & $\begin{array}{l}\text { Period effect } \\
\left(\mathrm{P} 1 / \mathrm{P} 3_{1}-\mathrm{P} 3_{6}\right)\end{array}$ & $\begin{array}{c}\text { Type } \times \text { Period } \\
\left(\mathrm{P} 1 / \mathrm{P}_{1}-\mathrm{P} 3_{6}\right) \\
\text { interaction }\end{array}$ \\
\hline \multicolumn{7}{|c|}{ Achilles vibration } \\
\hline \multirow[t]{2}{*}{ Speed } & $F(2,40)=54.9$ & $F(5,100)=36.3$ & $F(10,200)=10.3$ & $F(2,40)=80.6$ & $F(6,120)=114.1$ & $F(12,240)=38.9$ \\
\hline & $S$ & $S$ & $S$ & $S$ & $S$ & $S$ \\
\hline \multirow[t]{2}{*}{ AP position } & $F(2,40)=62.4$ & $F(5,100)=114.5$ & $F(10,200)=26.9$ & $F(2,40)=9.6$ & $F(6,120)=6.8$ & $F(12,240)=4.6$ \\
\hline & $S$ & $S$ & $S$ & $S$ & $S$ & $S$ \\
\hline \multirow[t]{2}{*}{ ML position } & $F(2,40)=45.3$ & $F(5,100)=3.0$ & $F(10,200)=35.7$ & $F(2,40)=9.60$ & $F(6,120)=0.8$ & $F(12,240)=7.6$ \\
\hline & $S$ & $S$ & $S$ & $S$ & $p=0.5$ & $S$ \\
\hline \multicolumn{7}{|c|}{ Peroneus vibration } \\
\hline \multirow[t]{2}{*}{ Speed } & $F(2,40)=35.9$ & $F(5,100)=13.8$ & $F(10,200)=6.1$ & $F(2,40)=12.9$ & $F(6,120)=24.8$ & $F(12,240)=7.6$ \\
\hline & $S$ & $S$ & $S$ & $S$ & $S$ & $S$ \\
\hline \multirow[t]{2}{*}{ AP position } & $F(2,40)=23.8$ & $F(5,100)=23.8$ & $F(10,250)=10.7$ & $F(2,40)=0.7$ & $F(6,120)=2.6$ & $F(12,240)=1.5$ \\
\hline & $S$ & $S$ & $S$ & $p=0.5$ & $S$ & $p=0.1$ \\
\hline \multirow[t]{2}{*}{ ML position } & $F(2,40)=0.7$ & $F(5,100)=0.1$ & $F(10,200)=1.6$ & $F(2,40)=0.2$ & $F(6,120)=0.3$ & $F(12,240)=0.6$ \\
\hline & $p=0.5$ & $p=1$ & $p=0.1$ & $p=0.8$ & $p=0.9$ & $p=0.9$ \\
\hline
\end{tabular}

ANOVA results carried out for each 'Tendon' (Achilles/Peroneus Longus) showing the effect of 'Type' and 'Period' for phases P2 and P3 of the trial and their interactions. Significant results are written in bold text. $S=$ statistically significant $(p<0.05)$. 


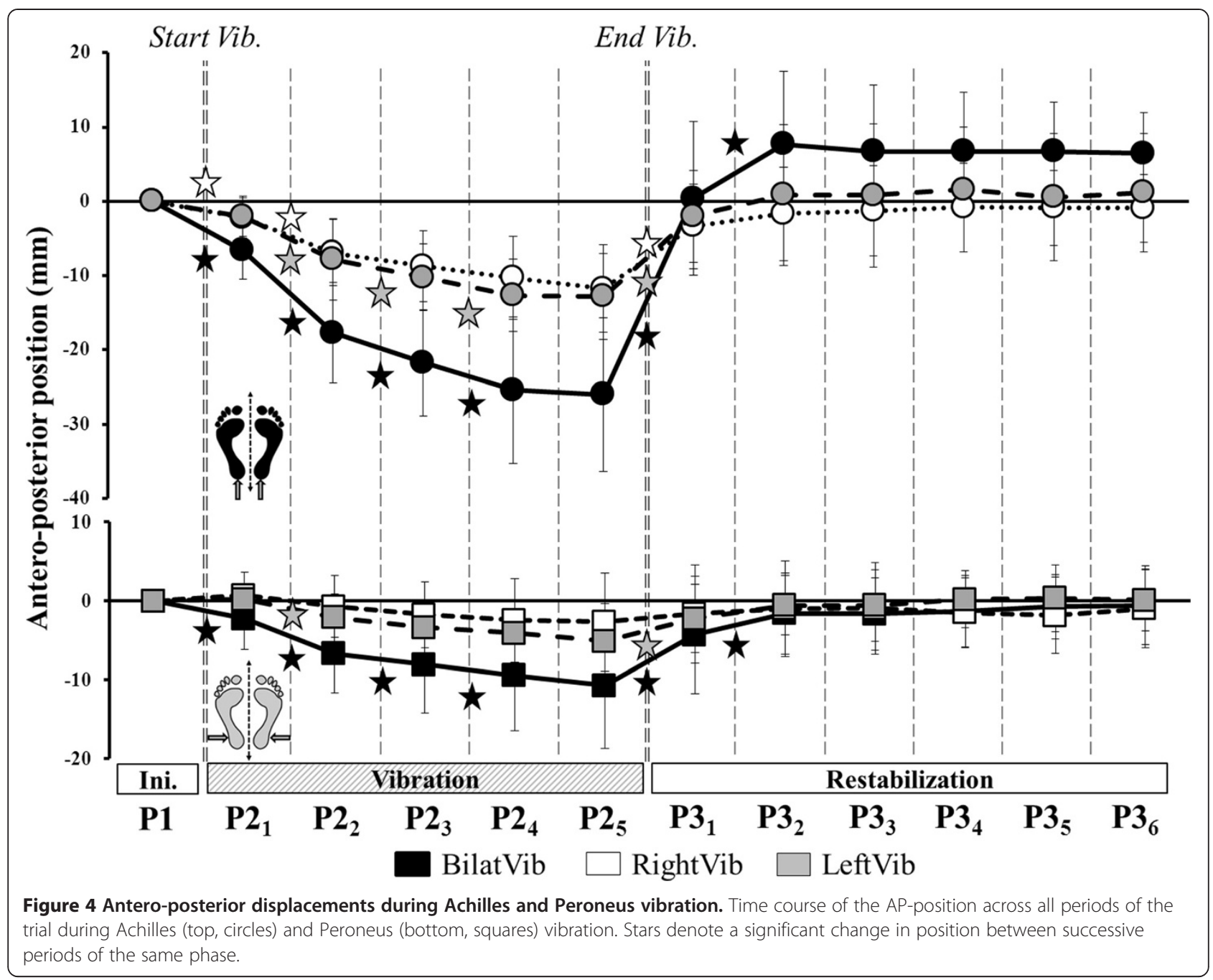

bilateral vibration and at $\mathrm{P} 3_{2}$ after unilateral vibration (Figure 6).

Extreme positions occurred very soon after the end of the vibrations (Figure 3). Cessation of vibration led to a maximal posterior position (AP-min), which was more posterior and occurred earlier after Achilles than Peroneus vibration. A maximal AP-position then occurred which was more anterior and earlier after Achilles than Peroneus vibration.

\section{Discussion}

Does the postural reaction depend on the plane controlled by the vibrated muscle?

The bilateral proprioceptive perturbation of both the Achilles and Peroneus tendons induced postural reactions in the sagittal plane but not in the frontal plane. All unilateral perturbations also induced reactions in the sagittal plane. Postural reactions occurred in the frontal plane only during unilateral Achilles vibration. The systematic antero-posterior reaction is in accordance with the functional roles of the muscles stimulated. The Triceps Surae muscle is massively implicated in the control of movement in the sagittal plane [5]. The role of Peroneus Longus in this plane has been little described but was expected since an illusory movement with a sagittal component has previously been demonstrated following vibration with the foot free [16]. These results show that, in order to maintain the system's requirements for balance and orientation in the sagittal plane, postural adjustments occurred immediately following the onset of the proprioceptive perturbation [26]. In this plane, the human musculoskeletal system has multiple degrees of freedom which have large ranges of motion [26]. It is very difficult for the postural system to counteract the effect of a proprioceptive perturbation without the use of visual input which usually stabilizes antero-posterior oscillations [27]. The amplitude of the destabilization may be related to the functional importance of the muscle in postural control. In the frontal plane, joint mobility is much more restricted [28] and the lower limbs form a closed-chain mechanical 


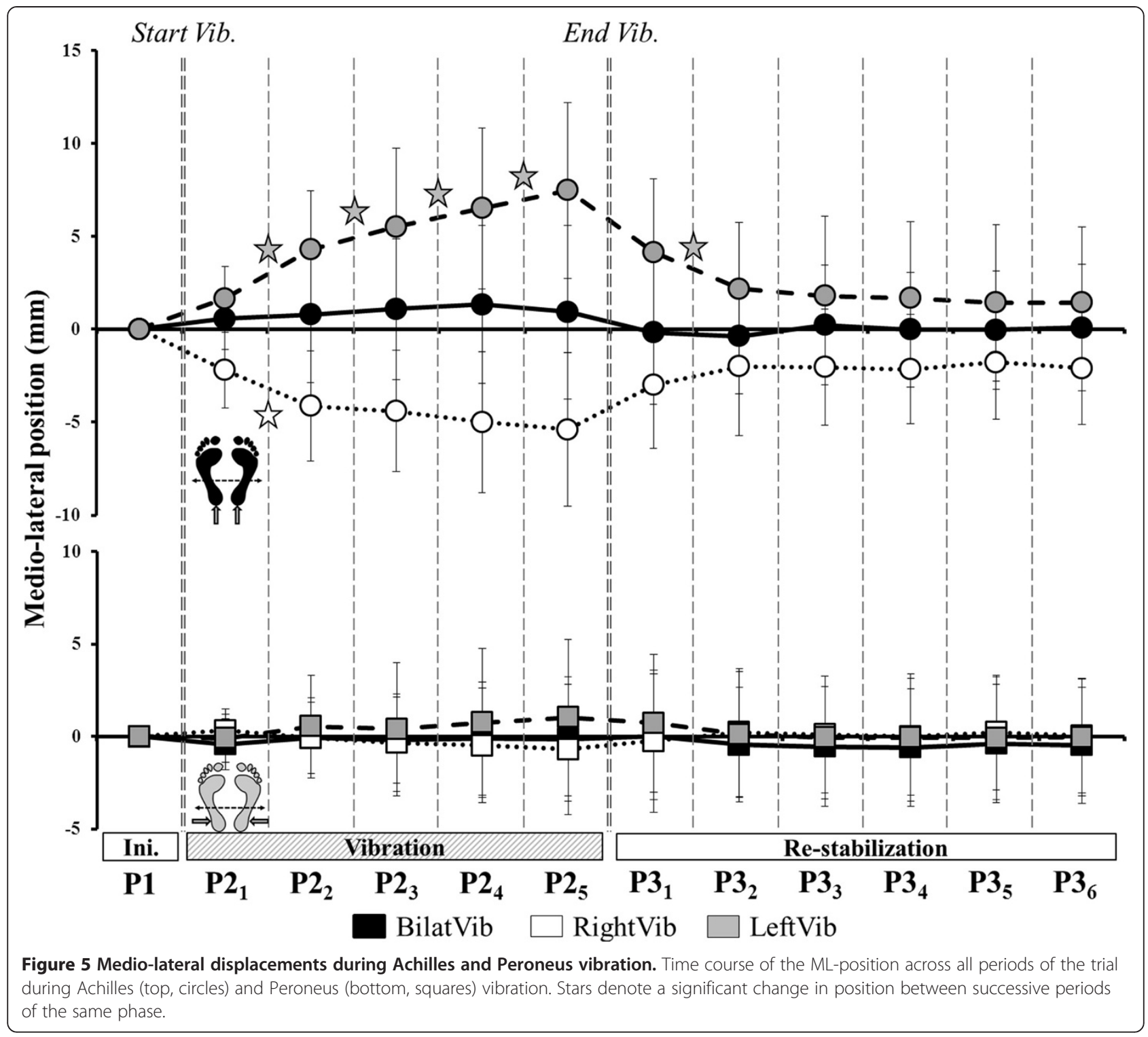

system [29] creating large biomechanical constraints. This could explain the lack of a significant medio-lateral displacement during the bilateral stimulation of both tendons. Some studies which used a constrained (such as toe-to-heel [30] which is highly sensitive to ML instability) or standardized foot position found a medio-lateral displacement in response to unilateral vibration on the lateral face of the ankle [3]. In the present study, the choice of a natural foot position could have created some mechanical medio-lateral stability [11]. In the normal standing position evaluated, the proprioceptive perturbation of the Peroneus was not sufficient to induce a medio-lateral displacement, despite the fact that it is directly implicated in the control of frontal plane motion. In contrast, the results showed that unilateral proprioceptive vibration of the Triceps Surae muscle always induced a medio-lateral displacement, despite the natural (stable) position of the feet. Thus, the medial displacement which occurred during unilateral Achilles vibration was not a general reaction to a single limb perturbation. The effect of the proprioceptive perturbation therefore seems to be influenced by the position of the vibrated muscle according to the planes of the musculoskeletal organization for normal standing control.

Is the role of the non-perturbed leg influenced by the direction of the induced movement?

The non-perturbed leg was not always sufficiently effective to ensure stability in the frontal plane. The postural system may adopt a voluntary or possibly reactive strategy, involving the transfer of body weight to the non-vibrated limb in order to avoid the perturbing effect of the vibrations. Nevertheless, the results of this study showed that 


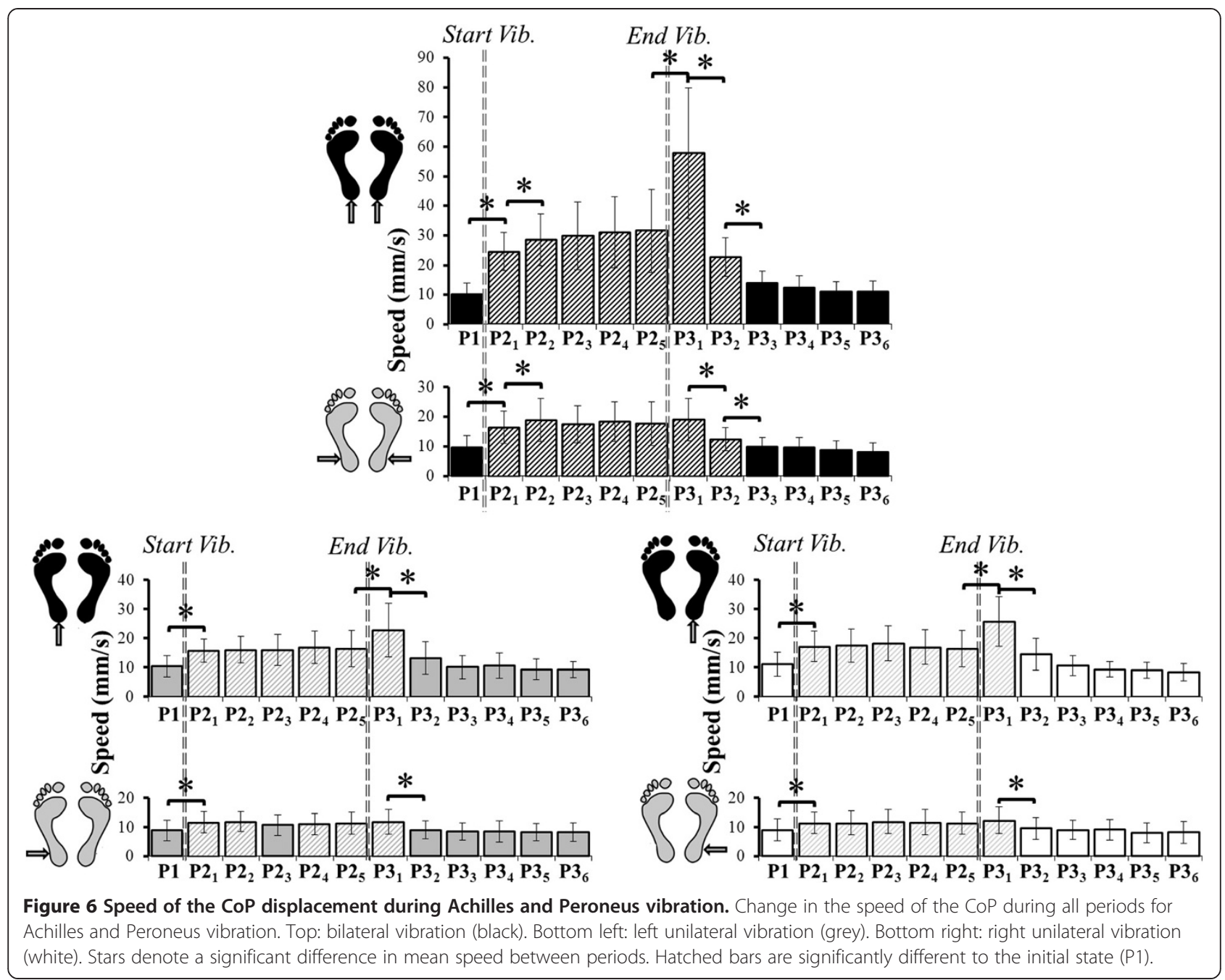

proprioceptive input from the non-perturbed leg did not completely prevent the antero-posterior displacement induced by vibration. However, analysis of the time course of the CoP displacement showed that its position was quickly stabilized. The halting of the CoP displacement may reflect a central phenomenon of sensory reweighting based on input from the non-vibrated limb. This process appeared to be effective for antero-posterior postural control but it failed to stabilize the medio-lateral axis. This highlights a paradox in the control of the different planes of body motion. Proprioceptive input from the non-perturbed leg did not prevent antero-posterior displacement but helped to gain stability over time; however, it did prevent mediolateral displacement. Nevertheless, if medio-lateral displacement began to occur, the postural system could not stop it. Multi-directional postural control is particularly complex in bipeds [31] and the postural system appears to be ineffective beyond a certain level of difficulty. The results of this study suggest that the level of difficulty is determined by the plane of the perturbation.
During tendon vibration, the CNS constructs a new sensory reference frame in order to maintain balance in spite of the illusion of falling forwards and the misperception of verticality [32]. When the proprioception of both Achilles tendons is simultaneously perturbed, the only available source of information regarding body position is vestibular. However, vestibular information is insufficient to restore the gravitational vertical without visual input if proprioceptive input is non-congruent. When the vibration stops, the initial postural referential must be restored. The initial antero-posterior position of the CoP was immediately restored after unilateral vibration of both muscles but not after bilateral Achilles vibration. The duration of the post-effect after bilateral vibration is, however, subject to discussion in the literature and appears to be highly subject-dependent $[19,33]$. In the present study, a post-effect was still present $25 \mathrm{~s}$ after the end of the $20 \mathrm{~s}$ of bilateral vibration. Thompson et al. [19] did not find any post-effect $25 \mathrm{~s}$ after the end of $30 \mathrm{~s}$ of bilateral vibration whereas Wierzbicka et al. [33] found some post-effects of 
bilateral vibration which persisted for up to $1 \mathrm{~h}$ in some subjects. However, a new finding of the present study was there was no significant post-effect after unilateral vibration. Thus, the non-perturbed limb clearly played an important role in the restoration of the postural referential, and this occurred as soon as the vibration ended. The results again showed the influence of the plane of motion controlled by the perturbed muscle. The position of the CoP remained shifted towards the contralateral limb following unilateral Achilles vibration. It could be hypothesized that (i) postural control in the frontal plane is not sufficiently accurate to detect this weight-bearing asymmetry; (ii) the postural abilities of young adults permit them to deal with this asymmetry without a risk of falling; (iii) the preceding perturbation may enhance a natural tendency for limb load asymmetry, suggested to be a functional adaptation for example to facilitate a step [32].

\section{Does the proprioceptive perturbation continue to disrupt} the system in spite of postural reorganization?

Schmid et al. [34] describe the process of adaptation to a perturbation of balance as the achievement of a steadystate, despite an ongoing perturbation. Antero-posterior displacement of the CoP during bilateral Achilles vibration is the main indicator of postural adaptation. Postural reactions which occur during vibration are not just the sum of local reflex adjustments [25] but are also the result of central changes. The position of the CoP was stable during $\mathrm{P} 2{ }_{4}-\mathrm{P} 2_{5}$ (12-16 $\mathrm{s}$ and $16-20 \mathrm{~s}$ of vibration). Its position during these periods was similar to the position recorded by Thompson et al. between 27.5 and $30 \mathrm{~s}$ [19]. This adaptive postural response may be an attempt to control the loss of stability caused by the multi-segmental displacement in response to the illusory movement. However, the antero-posterior stabilization also occurred following Peroneus vibration, despite the fact that the antero-posterior displacement of the CoP was smaller (less than that which occurred after 4-8 s of bilateral Achilles vibration). The assumption of mechanical stability does not explain this 2nd observation. The maximal posterior positions of the CoP occurred at similar times for all the vibration conditions (around 12-16 s of vibration), irrespective of the degree of disequilibrium. This is in accordance with the results of Schmid et al. [34] which showed that the adaptation process does not depend on the type of perturbation but rather on the time elapsed from the beginning of the perturbation. McKay et al. [22] found a similar phenomenon in children: the level of sensory-motor maturation affected the amplitude of the displacement induced by vibration, but not the time of occurrence of the maximal displacement. To our knowledge, studies which have analyzed the time course of postural effects only considered one type of vibration (either unilateral or bilateral). Capicíková et al.
[20] showed that the mean posterior displacement of the CoP during bilateral Achilles vibration increases with the duration of vibration (10, 20 and $30 \mathrm{~s}$ ) but the relationship is non-linear. The results of the present study showed that at least $16 \mathrm{~s}$ of vibration are necessary to induce most postural effects in young adults, regardless of the vibration condition. Less than $12 \mathrm{~s}$ of vibration $[35,36]$ is only sufficient to show the early effects of the proprioceptive perturbation. Results from studies involving stimulations of more than $20 \mathrm{~s}$ duration [3,19] show adaptive sensory-motor effects which are not always taken into account in the analysis. During vibration at the elbow joint, Cordo et al. [18] showed that the illusion of movement disappears at around $16 \mathrm{~s}$ of vibration, whereas the illusion of position persists. It therefore appears that the halting of the postural displacement around this time is the result of central sensory adaptations rather than biomechanical regulation. Thus, the duration of the vibration has to be taken into account in the interpretation of mean or final CoP positions and in the design and methodology of studies.

\section{Conclusions}

These results suggest that both the Triceps Surae and Peroneus muscles participate in the control of sagittal plane motion. The amplitude of the destabilization which occurs during vibration may be related to the functional role of the muscle in postural control. The proprioceptive perturbation of both the Triceps Surae and Peroneus Longus showed that proprioceptive information from these muscles plays a role in postural organization and integration within the motor pattern. Nevertheless, the results showed that the medial displacement of the CoP which occurred during unilateral Achilles vibration is not part of a general reaction to a single-limb perturbation. Proprioceptive input from the non-perturbed leg was not sufficient to avoid antero-posterior CoP displacement but helped to gain stability over time. This information could be useful for rehabilitation of the balance control process during walking. The results of this study showed that, even in a population of young subjects, 16 seconds of stimulation are necessary and sufficient to cause movement illusions and thus to destabilize subjects. These techniques could be helpful for the elaboration of therapeutic protocols.

\section{Abbreviations}

CoP: Centre of pressure; AP: Antero-posterior; ML: Medio-lateral.

\section{Competing interests}

The authors declare that they have no competing interests.

\section{Author information}

NCD is a physiotherapist and a PhD student. LM is a physiotherapist and the health manager of a center for neurological and orthopedic rehabilitation. JB is an associated professor. SM is a physiotherapist and an associated professor. 


\section{Authors' contributions}

NCD contributed to conception, experimental design, data analysis and interpretation, and write the manuscript. LM contributed to conception and subject recruitment. JB contributed to experimental design and critically revised the manuscript. SM contributed to conception, experimental design, data interpretation and help to write the manuscript. All authors read and approved the final manuscript.

\section{Acknowledgements}

This work was supported by the Paul Bennetot's Foundation. We thank J. Robertson for revising the English manuscript.

\section{Author details}

${ }^{1}$ Aix-Marseille Université, CNRS, ISM UMR 7287, 13288 Marseille, France. ${ }^{2}$ CRF Valmante, UGECAM-PACA, 13275 Marseille, France.

Received: 14 May 2014 Accepted: 15 August 2014

Published: 1 September 2014

\section{References}

1. Horak FB: Postural orientation and equilibrium: what do we need to know about neural control of balance to prevent falls? Age Ageing 2006, 35(Suppl 2):ii7-ii11.

2. Loram ID, Kelly SM, Lakie M: Human balancing of an inverted pendulum: is sway size controlled by ankle impedance? J Physiol 2001, 532(Pt 3):879-891.

3. Courtine G, De Nunzio AM, Schmid M, Beretta MV, Schieppati M: Stance- and locomotion-dependent processing of vibration-induced proprioceptive inflow from multiple muscles in humans. J Neurophysiol 2007, 97:772-779.

4. Gatev P, Thomas S, Kepple T, Hallett M: Feedforward ankle strategy of balance during quiet stance in adults. J Physiol 1999, 514:915-928.

5. Sozzi S, Honeine J-L, Do M-C, Schieppati M: Leg muscle activity during tandem stance and the control of body balance in the frontal plane. Clin Neurophysiol 2013, 124:1175-1186.

6. Grüneberg C, Duysens J, Honegger F, Allum JHJ: Spatio-temporal separation of roll and pitch balance-correcting commands in humans. J Neurophysiol 2005, 94:3143-3158.

7. De Ridder R, Willems T, De Mits S, Vanrenterghem J, Roosen P: Foot orientation affects muscle activation levels of ankle stabilizers in a single-legged balance board protocol. Hum Mov Sci 2014, 33:419-431.

8. Fitzpatrick R, McCloskey DI: Proprioceptive, visual and vestibular thresholds for the perception of sway during standing in humans. J Physiol 1994, 478:173-186.

9. Mcllroy WE, Bishop DC, Staines WR, Nelson AJ, Maki BE, Brooke JD: Modulation of afferent inflow during the control of balancing tasks using the lower limbs. Brain Res 2003, 961:73-80.

10. Bove M, Nardone A, Schieppati M: Effects of leg muscle tendon vibration on group la and group II reflex responses to stance perturbation in humans. J Physiol 2003, 550(Pt 2):617-630

11. Chiari L, Rocchi L, Cappello A: Stabilometric parameters are affected by anthropometry and foot placement. Clin Biomech (Bristol, Avon) 2002, 17:666-677.

12. Bok S-K, Lee TH, Lee SS: The effects of changes of ankle strength and range of motion according to aging on balance. Ann Rehabil Med 2013, 37:10-16.

13. Melzer I, Benjuya N, Kaplanski J: Postural stability in the elderly: a comparison between fallers and non-fallers. Age Ageing 2004, 33:602-607.

14. Henry SM, Fung J, Horak FB: Effect of Stance Width on Multidirectional Postural Responses. J Neurophysiol 2001, 85:559-570.

15. Roll JP, Bergenheim M, Ribot-Ciscar E: Proprioceptive population coding of two-dimensional limb movements in humans: II. Muscle-spindle feedback during "drawing-like" movements. Exp Brain Res 2000, 134:311-321.

16. Bergenheim M, Roll JP, Ribot-Ciscar E: Proprioceptive population coding of two-dimensional limb movements in humans: I. Muscle spindle feedback during spatially oriented movements. Exp Brain Res 2000, 134:301-310.

17. Roll J, Vedel J, Ribot E: Alteration of proprioceptive messages induced by tendon vibration in man: a microneurographic study. Exp Brain Res 1989, 76:213-222.

18. Cordo PJ, Gurfinkel VS, Brumagne S, Flores-Vieira C: Effect of slow, small movement on the vibration-evoked kinesthetic illusion. Exp Brain Res 2005, 167:324-334.
19. Thompson C, Bélanger M, Fung J: Effects of bilateral Achilles tendon vibration on postural orientation and balance during standing. Clin Neurophysiol 2007, 118:2456-2467.

20. Capicíková N, Rocchi L, Hlavacka F, Chiari L, Cappello A: Human postural response to lower leg muscle vibration of different duration. Physiol Res 2006, 55(Suppl 1):S129-S134.

21. Polónyová A, Hlavacka F: Human postural responses to different frequency vibrations of lower leg muscles. Physiol Res 2001, 50:405-410.

22. Mckay SM, Wu J, Angulo-barroso RM: Effect of Achilles Tendon Vibration on Posture in Children. Gait Posture 2014, 40:32-37.

23. Mcllroy WE, Maki BE: Preferred placement of the feet during quiet stance: development of a standardized foot placement for balance testing. Clin Biomech (Bristol, Avon) 1997, 12:66-70.

24. Ruget H, Blouin J, Coyle T, Mouchnino L: Modulation of proprioceptive inflow when initiating a step influences postural adjustments. Exp Brain Res 2010, 201:297-305.

25. Ivanenko YP, Talis VL, Kazennikov O: Support stability influences postural responses to muscle vibration in humans. Eur J Neurosci 1999, 11:647-654.

26. Massion J: Movement, Posture and Equilibrium: interaction and coordination. Prog Neurobiol 1992, 38:35-56.

27. Berencsi $\mathrm{A}$, Ishihara $\mathrm{M}$, Imanaka $\mathrm{K}$ : The functional role of central and peripheral vision in the control of posture. Hum Mov Sci 2005, 24:689-709.

28. Silfies S, Cholewicki J, Radebold A: The effects of visual input on postural control of the lumbar spine in unstable sitting. Hum Mov Sci 2003, 22:237-252.

29. Goodworth AD, Peterka RJ: Sensorimotor integration for multisegmental frontal plane balance control in humans. J Neurophysiol 2012, 107:12-28.

30. Lackner JR, Rabin E, Dizio P, Zelenin V, Saavedra SL, Teulier C, Smith BA, Kim $B$, Beutler BD, Martin BJ, Ulrich BD, Baccini M, Rinaldi LA, Federighi G, Vannucchi L, Paci M: Fingertip Contact Suppresses the Destabilizing Influence of Leg Muscle Vibration Fingertip Contact Suppresses the Destabilizing Influence of Leg Muscle Vibration. 2014:2217-2224.

31. Fransson P-A, Kristinsdottir E, Hafström A, Magnusson M, Johansson R: Balance control and adaptation during vibratory perturbations in middle-aged and elderly humans. Eur J Appl Physiol 2004, 91:595-603.

32. Barbieri G, Gissot A-S, Fouque F, Casillas J-M, Pozzo T, Pérennou D: Does proprioception contribute to the sense of verticality? Exp Brain Res 2008, 185:545-552.

33. Wierzbicka MM, Gilhodes JC, Roll JP: Vibration-Induced Postural Posteffects Vibration-Induced Postural Posteffects. J Neurophysiol 1998, 79:143-150

34. Schmid M, Bottaro A, Sozzi S, Schieppati M: Adaptation to continuous perturbation of balance: progressive reduction of postural muscle activity with invariant or increasing oscillations of the center of mass depending on perturbation frequency and vision conditions. Hum Mov Sci 2011, 30:262-278

35. Abrahámová D, Mancini M, Hlavacka F, Chiari L: The age-related changes of trunk responses to Achilles tendon vibration. Neurosci Lett 2009, 467:220-224.

36. Barbieri G, Gissot A-S, Nougier V, Pérennou D: Achilles tendon vibration shifts the center of pressure backward in standing and forward in sitting in young subjects. Clin Neurophysiol 2013, 43:237-242.

\section{doi:10.1186/1743-0003-11-130}

Cite this article as: Duclos et al:: Postural stabilization during bilateral and unilateral vibration of ankle muscles in the sagittal and frontal planes. Journal of NeuroEngineering and Rehabilitation 2014 11:130. 